Research Article

\title{
Hesitant Fuzzy Soft Set and Its Applications in Multicriteria Decision Making
}

\author{
Fuqiang Wang, Xihua Li, and Xiaohong Chen \\ School of Business, Central South University, Changsha, Hunan 410083, China \\ Correspondence should be addressed to Xihua Li; 251560913@qq.com
}

Received 16 April 2014; Accepted 16 June 2014; Published 3 July 2014

Academic Editor: Zhenfu Cao

Copyright (c) 2014 Fuqiang Wang et al. This is an open access article distributed under the Creative Commons Attribution License, which permits unrestricted use, distribution, and reproduction in any medium, provided the original work is properly cited.

\begin{abstract}
Molodtsov's soft set theory is a newly emerging mathematical tool to handle uncertainty. However, the classical soft sets are not appropriate to deal with imprecise and fuzzy parameters. This paper aims to extend the classical soft sets to hesitant fuzzy soft sets which are combined by the soft sets and hesitant fuzzy sets. Then, the complement, "AND", "OR", union and intersection operations are defined on hesitant fuzzy soft sets. The basic properties such as DeMorgan's laws and the relevant laws of hesitant fuzzy soft sets are proved. Finally, with the help of level soft set, the hesitant fuzzy soft sets are applied to a decision making problem and the effectiveness is proved by a numerical example.
\end{abstract}

\section{Introduction}

In the real world, there are many complicated problems in economics, engineering, environment, social science, and management science. They are characterized with uncertainty, imprecision, and vagueness. We cannot successfully utilize the classical methods to deal with these problems because there are various types of uncertainties involved in these problems. Moreover, though there are many theories, such as theory of probability, theory of fuzzy sets, theory of interval mathematics, and theory of rough sets to be considered as mathematical tools to deal with uncertainties, Molodtsov [1] pointed out all these theories had their own limitations. Moreover, in order to overcome these difficulties, Molodtsov [1] firstly proposed a new mathematical tool named soft set theory to deal with uncertainty and imprecision. This theory has been demonstrated to be a useful tool in many applications such as decision making, measurement theory, and game theory.

The soft set model can be combined with other mathematical models. Maji et al. [2] firstly presented the concept of fuzzy soft set by combining the theories of fuzzy set and soft set together. Furthermore, Maji et al. [3-5] established the notion of intuitionistic fuzzy soft set which was based on a combination of the intuitionistic fuzzy set $[6,7]$ and soft set models. By combining the interval-valued fuzzy set $[8,9]$ and soft set, Yang et al. [10] presented the concept of the intervalvalued fuzzy soft set. Jiang et al. [11] initiated the concept of interval-valued intuitionistic fuzzy soft sets as an intervalvalued fuzzy extension of the intuitionistic fuzzy soft set theory or an intuitionistic fuzzy extension of the interval-valued fuzzy soft set theory. Recently, Xiao et al. [12] presented the trapezoidal fuzzy soft set and Yang et al. [13] introduced the multifuzzy soft set, respectively, and applied them in decision making problems. Roy and Maji [14], Kong et al. [15], Feng et al. [16], and Jiang et al. [17] also applied the fuzzy soft set in the decision making problems. Jun [18] initiated the application of soft sets in BCK/BCI-algebras and introduced the concept of soft BCK/BCI-algebras. Furthermore, Jun and Park [19] and Jun et al. [20, 21] applied the soft sets in ideal theory of BCK/BCI-algebras and d-algebras. Feng et al. [22, 23] initiated the concept of rough soft sets, soft rough sets, and soft rough fuzzy sets.

Recently, in order to tackle the difficulty in establishing the degree of membership of an element in a set, Torra and Narukawa [24] and Torra [25] proposed the concept of a hesitant fuzzy set. This new extension of fuzzy set can handle the cases that the difficulty in establishing the membership 
degree does not arise from a margin of error (as in intuitionistic or interval-valued fuzzy sets) or a specified possibility distribution of the possible values (as in type-2 fuzzy sets), but arises from our hesitation among a few different values [26]. Thus the hesitant fuzzy set can more accurately reflect the people's hesitancy in stating their preferences over objects, compared to the fuzzy set and its many classical extensions. The purpose of this paper is to extend the soft set model to the hesitant fuzzy set, and, thus, we establish a new soft set model named hesitant fuzzy soft set.

The rest of this paper is organized as follows. We first review some background on soft set, fuzzy soft set, and hesitant fuzzy set in Section 2. In Section 3, the concepts and operations of hesitant fuzzy soft set are proposed and their properties are discussed in detail. In Section 4, we apply the proposed hesitant fuzzy soft set to a decision making problem and give an explicit algorithm. Finally, we conclude in Section 5 .

\section{Preliminaries}

2.1. Soft Sets. Suppose that $U$ is an initial universe set, $E$ is a set of parameters, $P(U)$ is the power set of $U$ and $A \subset E$.

Definition 1 (see [1]). A pair $(F, A)$ is called a soft set over $U$, where $F$ is a mapping given by $F: A \rightarrow P(U)$.

In other words, a soft set over $U$ is a parameterized family of subsets of the universe $U$. For $e \in A, F(e)$ may be considered as the set of $e$-approximate elements of the soft set $(F, A)$.

Example 2. Suppose that $U=\left\{h_{1}, h_{2}, h_{3}, h_{4}, h_{5}, h_{6}\right\}$ is a set of houses and $A=\left\{e_{1}, e_{2}, e_{3}, e_{4}, e_{5}\right\}$ is a set of parameters, which stands for the parameters "cheap," "beautiful," "size," "location," and "surrounding environment," respectively. In this case, a soft set $(F, A)$ can be defined as a mapping from parameter set $A$ to the set of all subsets of $U$. In this way, the set of the houses with specific characteristics can be described.

Assume that $F\left(e_{1}\right)=\left\{h_{2}, h_{4}\right\}, F\left(e_{2}\right)=\left\{h_{1}, h_{3}\right\}, F\left(e_{3}\right)=$ $\left\{h_{3}, h_{4}, h_{5}\right\}, F\left(e_{4}\right)=\left\{h_{1}, h_{3}, h_{5}\right\}$, and $F\left(e_{5}\right)=\left\{h_{2}\right\}$, which mean "houses (cheap)" whose function-value is the set $\left\{h_{2}, h_{4}\right\}$, "houses (beautiful)" whose function-value is the set $\left\{h_{1}, h_{3}\right\}$, "houses (big size)" whose function-value is the set $\left\{h_{3}, h_{4}, h_{5}\right\}$, "houses (in good location)" whose function-value is the set $\left\{h_{1}, h_{3}, h_{5}\right\}$, "houses (great surrounding environment)" whose function-value is the set $\left\{h_{2}\right\}$, respectively. Then we can view the soft set $(F, A)$ as consisting of the following collection of approximations:

$$
\begin{gathered}
(F, A)=\left\{\left(e_{1},\left\{h_{2}, h_{4}\right\}\right),\left(e_{2},\left\{h_{1}, h_{3}\right\}\right),\left(e_{3},\left\{h_{3}, h_{4}, h_{5}\right\}\right),\right. \\
\left.\left(e_{4},\left\{h_{1}, h_{3}, h_{5}\right\}\right),\left(e_{5},\left\{h_{2}\right\}\right)\right\} .
\end{gathered}
$$

For the purpose of storing a soft set in a computer, we can represent the soft set defined in Example 2 in tabular form as Table 1 shows.
TABLE 1: The tabular representation of the soft set $(F, A)$.

\begin{tabular}{lccccc}
\hline$U$ & "Cheap" "Beautiful" & "Size" "Location" & $\begin{array}{c}\text { "Great } \\
\text { surrounding } \\
\text { environment" }\end{array}$ \\
\hline$h_{1}$ & 0 & 1 & 0 & 1 & 0 \\
$h_{2}$ & 1 & 0 & 0 & 0 & 1 \\
$h_{3}$ & 0 & 1 & 1 & 1 & 0 \\
$h_{4}$ & 1 & 0 & 1 & 0 & 0 \\
$h_{5}$ & 0 & 0 & 1 & 1 & 0 \\
$h_{6}$ & 0 & 0 & 0 & 0 & 0 \\
\hline
\end{tabular}

TABLE 2: The tabular representation of the fuzzy soft set $(\widetilde{F}, A)$.

\begin{tabular}{cccccc}
\hline$U$ & "Cheap" “Beautiful" & "Size" "Location" & $\begin{array}{c}\text { "Great } \\
\text { surrounding } \\
\text { environment" }\end{array}$ \\
\hline$h_{1}$ & 0.2 & 0.6 & 0.4 & 0.3 & 0.6 \\
$h_{2}$ & 0.5 & 0.5 & 0.6 & 0.2 & 0.3 \\
$h_{3}$ & 0.3 & 0.6 & 0.8 & 0.5 & 0.5 \\
$h_{4}$ & 0.3 & 0.7 & 0.3 & 0.7 & 0.4 \\
$h_{5}$ & 0.4 & 0.4 & 0.4 & 0.5 & 0.7 \\
$h_{6}$ & 0.6 & 0.3 & 0.7 & 0.8 & 0.3 \\
\hline
\end{tabular}

\subsection{Fuzzy Soft Sets}

Definition 3 (see [2]). Let $\widetilde{P}(U)$ be the set of all fuzzy subsets of $U$, a pair $(\widetilde{F}, A)$ is called a fuzzy soft set over $U$, where $\widetilde{F}$ is a mapping given by $\widetilde{F}: A \rightarrow \widetilde{P}(U)$.

Example 4. Reconsider Example 2. In real life, the perception of the people is characterized by a certain degree of vagueness and imprecision; thus, when people consider if a house $h_{1}$ is cheap the information cannot be expressed with only two crisp numbers 0 and 1 . Instead it should be characterized by a membership function $\mu_{\widetilde{A}}(x)$ which associates with each element a real number in the interval $[0,1]$. Then, fuzzy soft set $(\widetilde{F}, A)$ can describe the characteristics of the house under the fuzzy information.

$$
\begin{aligned}
& \widetilde{F}\left(e_{1}\right)=\left\{\frac{h_{1}}{0.2}, \frac{h_{2}}{0.5}, \frac{h_{3}}{0.3}, \frac{h_{4}}{0.3}, \frac{h_{5}}{0.4}, \frac{h_{6}}{0.6}\right\}, \\
& \widetilde{F}\left(e_{2}\right)=\left\{\frac{h_{1}}{0.6}, \frac{h_{2}}{0.5}, \frac{h_{3}}{0.6}, \frac{h_{4}}{0.7}, \frac{h_{5}}{0.4}, \frac{h_{6}}{0.3}\right\}, \\
& \widetilde{F}\left(e_{3}\right)=\left\{\frac{h_{1}}{0.4}, \frac{h_{2}}{0.6}, \frac{h_{3}}{0.8}, \frac{h_{4}}{0.3}, \frac{h_{5}}{0.4}, \frac{h_{6}}{0.7}\right\}, \\
& \widetilde{F}\left(e_{4}\right)=\left\{\frac{h_{1}}{0.3}, \frac{h_{2}}{0.2}, \frac{h_{3}}{0.5}, \frac{h_{4}}{0.7}, \frac{h_{5}}{0.5}, \frac{h_{6}}{0.8}\right\}, \\
& \widetilde{F}\left(e_{5}\right)=\left\{\frac{h_{1}}{0.6}, \frac{h_{2}}{0.3}, \frac{h_{3}}{0.5}, \frac{h_{4}}{0.4}, \frac{h_{5}}{0.7}, \frac{h_{6}}{0.3}\right\} .
\end{aligned}
$$

Similarly, for the purpose of storing a fuzzy soft set in a computer, we could also represent the fuzzy soft set defined in Example 4 in Table 2. 


\subsection{Hesitant Fuzzy Sets}

Definition 5 (see [25]). A hesitant fuzzy set (HFS) on $U$ is in terms of a function that when applied to $U$ returns a subset of $[0,1]$, which can be represented as the following mathematical symbol:

$$
\widetilde{A}=\left\{\left\langle u, h_{\widetilde{A}}(u)\right\rangle \mid u \in U\right\}
$$

where $h_{\widetilde{A}}(u)$ is a set of values in $[0,1]$, denoting the possible membership degrees of the element $u \in U$ to the set $\widetilde{A}$. For convenience, we call $h_{\widetilde{A}}(u)$ a hesitant fuzzy element (HFE) and $H$ the set of all HFEs.

Furthermore, Torra [25] defined the empty hesitant set and the full hesitant set.

Definition 6 (see [25]). Given hesitant fuzzy set $\widetilde{A}$, if $h(u)=$ $\{0\}$ for all $u$ in $U$, then $\widetilde{A}$ is called the null hesitant fuzzy set, denoted by $\widetilde{\phi}$. If $h(u)=\{1\}$ for all $u$ in $U$, then $\widetilde{A}$ is called the full hesitant fuzzy set, denoted by $\widetilde{1}$.

Definition 7 (see [27]). For a HFE $h, s(h)=(1 / l(h)) \sum_{\gamma \in h} \gamma$ is called the score function of $h$, where $l(h)$ is the number of the values in $h$. For two HFEs $h_{1}$ and $h_{2}$, if $s\left(h_{1}\right)>s\left(h_{2}\right)$, then $h_{1}>h_{2}$; if $s\left(h_{1}\right)=s\left(h_{2}\right)$, then $h_{1}=h_{2}$.

Let $h_{1}$ and $h_{2}$ be two HFEs. It is noted that the number of values in different HFEs $h_{1}$ and $h_{2}$ are commonly different; that is, $l\left(h_{1}\right) \neq l\left(h_{2}\right)$. For convenience, let $l=\max \left\{l\left(h_{1}\right), l\left(h_{2}\right)\right\}$. To operate correctly, we should extend the shorter one until both of them have the same length when we compare them. To extend the shorter one, the best way is to add the same value several times in it [28]. In fact, we can extend the shorter one by adding any value in it. The selection of this value mainly depends on the decision makers' risk preferences. Optimists anticipate desirable outcomes and may add the maximum value, while pessimists expect unfavorable outcomes and may add the minimum value. For example, let $h_{1}=\{0.1,0.2,0.3\}$, let $h_{2}=\{0.4,0.5\}$, and let $l\left(h_{1}\right)>l\left(h_{2}\right)$. To operate correctly, we should extend $h_{2}$ to $h_{2}^{\prime}=\{0.4,0.4,0.5\}$ until it has the same length of $h_{1}$, the optimist may extend $h_{2}$ as $h_{2}^{\prime}=\{0.4,0.5,0.5\}$ and the pessimist may extend it as $h_{2}^{\prime}=\{0.4,0.4,0.5\}$. Although the results may be different if we extend the shorter one by adding different values, this is reasonable because the decision makers' risk preferences can directly influence the final decision. The same situation can also be found in many existing references [29-31]. In this paper, we assume that the decision makers are all pessimistic (other situations can be studied similarly).

We arrange the elements in $h_{\widetilde{A}}(u)$ in decreasing order, and let $h_{\widetilde{A}}^{\sigma(j)}(u)$ be the $j$ th largest value in $h_{\widetilde{A}}(u)$.

Definition 8. Given two hesitant fuzzy sets $\widetilde{M}=\left\{\left\langle u, h_{\widetilde{M}}(u)\right\rangle \mid\right.$ $u \in U\}$ and $\widetilde{N}=\left\{\left\langle u, h_{\widetilde{N}}(u)\right\rangle \mid u \in U\right\}, \widetilde{M}$ is called the fuzzy subset of $\widetilde{N}$ if and only if $h_{M}^{\sigma(j)}(u) \leq h_{N}^{\sigma(j)}(u)$, for $\forall u \in U$ and $j=1,2, \ldots, l$, which can be denoted by $\widetilde{M} \widetilde{\subseteq} \widetilde{N}$.
Definition 9. $\widetilde{M}$ and $\widetilde{N}$ are two hesitant fuzzy sets, we call $\widetilde{M}$ and $\widetilde{N}$ is hesitant fuzzy equal if and only if

(1) $\widetilde{M} \widetilde{\widetilde{N}}$

(2) $\widetilde{M} \supseteq \widetilde{N}$

which can be denoted by $\widetilde{M}=\widetilde{N}$.

Given three HFEs, $h, h_{1}$, and $h_{2}$, Torra [25] and Torra and Narukawa [24] defined the following HFE operations:
(1) $h^{c}=\cup_{\gamma \in h}\{1-\gamma\}$,
(2) $h_{1} \cup h_{2}=\left\{h \in\left(h_{1} \cup h_{2}\right) \mid h \geq \max \left(h_{1}^{-}, h_{2}^{-}\right)\right\}$,
(3) $h_{1} \cap h_{2}=\left\{h \in\left(h_{1} \cup h_{2}\right) \mid h \leq \min \left(h_{1}^{+}, h_{2}^{+}\right)\right\}$,

where $h^{-}(u)=\min h(u)$ and $h^{+}(u)=\max h(u)$ are the lower bound and upper bound of the given hesitant fuzzy elements, respectively.

Therefore, giving two hesitant fuzzy soft sets $\widetilde{A}$ and $\widetilde{B}$, we can define the following operations:

(1) $\widetilde{A}^{c}=\left\{\left\langle u, h_{\widetilde{A}}^{c}(u)\right\rangle \mid u \in U\right\}$;

(2) $\widetilde{A} \cup \widetilde{B}=\left\{\left\langle u, h_{\widetilde{A}}(u) \cup h_{\widetilde{B}}(u)\right\rangle \mid u \in U\right\}$;

(3) $\widetilde{A} \cap \widetilde{B}=\left\{\left\langle u, h_{\widetilde{A}}(u) \cap h_{\widetilde{B}}(u)\right\rangle \mid u \in U\right\}$.

Moreover, for the aggregation of hesitant fuzzy information, Xia and Xu [27] defined the following new operations on HFEs $h, h_{1}$, and $h_{2}$ :
(1) $h^{\lambda}=\cup_{\gamma \in h}\left\{\gamma^{\lambda}\right\}$;
(2) $\lambda h=\cup_{\gamma \in h}\left\{1-(1-\gamma)^{\lambda}\right\}$;
(3) $h_{1} \oplus h_{2}=\cup_{\gamma_{1} \in h_{1}, \gamma_{2} \in h_{2}}\left\{\gamma_{1}+\gamma_{2}-\gamma_{1} \gamma_{2}\right\}$;
(4) $h_{1} \otimes h_{2}=\cup_{\gamma_{1} \in h_{1}, \gamma_{2} \in h_{2}}\left\{\gamma_{1} \gamma_{2}\right\}$.

\section{Hesitant Fuzzy Soft Sets}

\subsection{The Concept of Hesitant Fuzzy Soft Sets}

Definition 10. Let $\widetilde{H}(U)$ be the set of all hesitant fuzzy sets in $U$; a pair $(\widetilde{F}, A)$ is called a hesitant fuzzy soft set over $U$, where $\widetilde{F}$ is a mapping given by

$$
\widetilde{F}: A \longrightarrow \widetilde{H}(U) \text {. }
$$

A hesitant fuzzy soft set is a mapping from parameters to $\widetilde{H}(U)$. It is a parameterized family of hesitant fuzzy subsets of $U$. For $e \in A, \widetilde{F}(e)$ may be considered as the set of $e$ approximate elements of the hesitant fuzzy soft set $(\widetilde{F}, A)$.

Example 11. Continue to consider Example 2. Mr. X evaluates the optional six houses under various attributes with hesitant fuzzy element; then, hesitant fuzzy soft set $(\widetilde{F}, A)$ can describe the characteristics of the house under the hesitant fuzzy information. 
TABLE 3: The tabular representation of the hesitant fuzzy soft set $(\widetilde{F}, A)$.

\begin{tabular}{lccccc}
\hline$U$ & "Cheap" & "Beautiful" & "Size" & "Location" & "Great surrounding environment" \\
\hline$h_{1}$ & $\{0.2,0.3\}$ & $\{0.4,0.6,0.7\}$ & $\{0.2,0.4\}$ & $\{0.3,0.5,0.6\}$ & $\{0.6\}$ \\
$h_{2}$ & $\{0.5,0.6\}$ & $\{0.5,0.7,0.8\}$ & $\{0.6,0.7\}$ & $\{0.2\}$ & $\{0.2,0.3,0.5\}$ \\
$h_{3}$ & $\{0.3\}$ & $\{0.6,0.8\}$ & $\{0.8,0.9\}$ & $\{0.5\}$ & $\{0.5,0.7\}$ \\
$h_{4}$ & $\{0.3,0.5\}$ & $\{0.7,0.9\}$ & $\{0.3,0.5\}$ & $\{0.6,0.7\}$ & $\{0.2,0.4\}$ \\
$h_{5}$ & $\{0.4,0.5\}$ & $\{0.3,0.4,0.5\}$ & $\{0.4,0.6\}$ & $\{0.5,0.6\}$ & $\{0.5,0.7\}$ \\
$h_{6}$ & $\{0.6,0.7\}$ & $\{0.3\}$ & $\{0.7\}$ & $\{0.8\}$ & $\{0.3,0.5\}$ \\
\hline
\end{tabular}

Consider

$$
\begin{aligned}
& \widetilde{F}\left(e_{1}\right)=\left\{\frac{h_{1}}{\{0.2,0.3\}}, \frac{h_{2}}{\{0.5,0.6\}}, \frac{h_{3}}{\{0.3\}}, \frac{h_{4}}{\{0.3,0.5\}},\right. \\
& \left.\frac{h_{5}}{\{0.4,0.5\}}, \frac{h_{6}}{\{0.6,0.7\}}\right\} \text {, } \\
& \widetilde{F}\left(e_{2}\right)=\left\{\frac{h_{1}}{\{0.4,0.6,0.7\}}, \frac{h_{2}}{\{0.5,0.7,0.8\}}, \frac{h_{3}}{\{0.6,0.8\}},\right. \\
& \left.\frac{h_{4}}{\{0.7,0.9\}}, \frac{h_{5}}{\{0.3,0.4,0.5\}}, \frac{h_{6}}{\{0.3\}}\right\} \text {, } \\
& \widetilde{F}\left(e_{3}\right)=\left\{\frac{h_{1}}{\{0.2,0.4\}}, \frac{h_{2}}{\{0.6,0.7\}}, \frac{h_{3}}{\{0.8,0.9\}}, \frac{h_{4}}{\{0.3,0.5\}},\right. \\
& \left.\frac{h_{5}}{\{0.4,0.6\}}, \frac{h_{6}}{\{0.7\}}\right\} \text {, } \\
& \widetilde{F}\left(e_{4}\right)=\left\{\frac{h_{1}}{\{0.3,0.5,0.6\}}, \frac{h_{2}}{\{0.2\}}, \frac{h_{3}}{\{0.5\}}, \frac{h_{4}}{\{0.6,0.7\}},\right. \\
& \left.\frac{h_{5}}{\{0.5,0.6\}}, \frac{h_{6}}{\{0.8\}}\right\} \\
& \widetilde{F}\left(e_{5}\right)=\left\{\frac{h_{1}}{\{0.6\}}, \frac{h_{2}}{\{0.2,0.3,0.5\}}, \frac{h_{3}}{\{0.5,0.7\}}, \frac{h_{4}}{\{0.2,0.4\}},\right. \\
& \left.\frac{h_{5}}{\{0.5,0.7\}}, \frac{h_{6}}{\{0.3,0.5\}}\right\} \text {. }
\end{aligned}
$$

Similarly, we can also represent the hesitant fuzzy soft set in the form of Table 3 for the purpose of storing the hesitant fuzzy soft set in a computer.

Definition 12. Let $A, B \in E .(\widetilde{F}, A)$ and $(\widetilde{G}, B)$ are two hesitant fuzzy soft sets over $U$. $(\widetilde{F}, A)$ is said to be a hesitant fuzzy soft subset of $(\widetilde{G}, B)$ if

(1) $A \subseteq B$,

(2) For all $e \in A, \widetilde{F}(e) \widetilde{\subseteq} \widetilde{G}(e)$.

In this case, we write $(\widetilde{F}, A) \widetilde{\subseteq}(\widetilde{G}, B)$.

Example 13. Given two hesitant fuzzy soft sets $(\widetilde{F}, A)$ and $(\widetilde{G}, B), U=\left\{h_{1}, h_{2}, h_{3}, h_{4}, h_{5}, h_{6}\right\}$ is the set of the optional houses. $A=\left\{e_{1}, e_{2}\right\}=\{$ cheap, beautiful $\}, B=\left\{e_{1}, e_{2}, e_{3}\right\}=$ \{cheap, beautiful, size $\}$, and

$$
\begin{aligned}
\widetilde{F}\left(e_{1}\right)= & \left\{\frac{h_{1}}{\{0.2,0.3\}}, \frac{h_{2}}{\{0.5,0.6\}}, \frac{h_{3}}{\{0.3\}}, \frac{h_{4}}{\{0.3,0.5\}},\right. \\
& \left.\frac{h_{5}}{\{0.4,0.5\}}, \frac{h_{6}}{\{0.6,0.7\}}\right\}, \\
\widetilde{F}\left(e_{2}\right)= & \left\{\frac{h_{1}}{\{0.4,0.6,0.7\}}, \frac{h_{2}}{\{0.5,0.7,0.8\}}, \frac{h_{3}}{\{0.6,0.8\}},\right. \\
\widetilde{G}\left(e_{1}\right)= & \left\{\frac{h_{4}}{\{0.7,0.9\}}, \frac{h_{1}}{\{0.3,0.4,0.5\}}, \frac{h_{2}}{\{0.6,0.8\}}, \frac{h_{3}}{\{0.4,0.5\}}, \frac{h_{4}}{\{0.3,0.4,0.5\}},\right. \\
\widetilde{G}\left(e_{2}\right)= & \left\{\frac{h_{5}}{\{0.5\}}, \frac{h_{6}}{\{0.7\}}\right\}, \\
\widetilde{G}\left(e_{3}\right)= & \left\{\frac{h_{1}}{\{0.2,0.4\}}, \frac{h_{1}}{\{0.6,0.7\}}, \frac{h_{3}}{\{0.8,0.9\}}, \frac{h_{4}}{\{0.3,0.5\}},\right. \\
\left.\left.\frac{\{0.4,0.6\}}{\{0.8,0.9\}}, \frac{h_{4}}{\{0.7\}}\right\} ., \frac{h_{5}}{\{0.5\}}, \frac{h_{6}}{\{0.5\}}\right\}, & \frac{h_{2}}{\{0.7,0.8\}}, \frac{h_{3}}{\{0.9\}},
\end{aligned}
$$

Then, we have $(\widetilde{F}, A) \widetilde{\subseteq}(\widetilde{G}, B)$.

Definition 14. Two hesitant fuzzy soft sets $(\widetilde{F}, A)$ and $(\widetilde{G}, B)$ are said to be hesitant fuzzy soft equal if $(\widetilde{F}, A)$ is a hesitant fuzzy soft subset of $(\widetilde{G}, B)$ and $(\widetilde{G}, B)$ is a hesitant fuzzy soft subset of $(\widetilde{F}, A)$.

In this case, we write $(\widetilde{F}, A) \cong(\widetilde{G}, B)$.

Definition 15. A hesitant fuzzy soft set $(\widetilde{F}, A)$ is said to be empty hesitant fuzzy soft set, denoted by $\widetilde{\Phi}_{A}$, if $\widetilde{F}(e)=\widetilde{\phi}$ for all $e \in A$.

Definition 16. A hesitant fuzzy soft set $(\widetilde{F}, A)$ is said to be full hesitant fuzzy soft set, denoted by $\widetilde{U}_{A}$, if $\widetilde{F}(e)=\widetilde{1}$ for all $e \in A$. 
TABLE 4: The result of "AND" operation on $(\widetilde{F}, A)$ and $(\widetilde{G}, B)$.

\begin{tabular}{ccccccc}
\hline$U$ & $e_{1}, e_{1}$ & $e_{1}, e_{2}$ & $e_{1}, e_{3}$ & $e_{2}, e_{1}$ & $e_{2}, e_{2}$ & $e_{2}, e_{3}$ \\
\hline$h_{1}$ & $\{0.2,0.3\}$ & $\{0.2,0.3\}$ & $\{0.2,0.3\}$ & $\{0.3\}$ & $\{0.4,0.5,0.6,0.7\}$ & $\{0.2,0.4\}$ \\
$h_{2}$ & $\{0.5,0.6\}$ & $\{0.5,0.6\}$ & $\{0.5,0.6\}$ & $\{0.5,0.6,0.7,0.8\}$ & $\{0.5,0.7,0.8\}$ & $\{0.5,0.6,0.7\}$ \\
$h_{3}$ & $\{0.3\}$ & $\{0.3\}$ & $\{0.3\}$ & $\{0.4,0.5\}$ & $\{0.6,0.8\}$ & $\{0.6,0.8\}$ \\
$h_{4}$ & $\{0.3,0.5\}$ & $\{0.3,0.5\}$ & $\{0.3,0.5\}$ & $\{0.3,0.4,0.5\}$ & $\{0.7,0.8,0.9\}$ & $\{0.3,0.5\}$ \\
$h_{5}$ & $\{0.3,0.4,0.5\}$ & $\{0.4,0.5\}$ & $\{0.4,0.5\}$ & $\{0.3,0.4,0.5\}$ & $\{0.3,0.4,0.5\}$ & $\{0.3,0.4,0.5\}$ \\
$h_{6}$ & $\{0.3\}$ & $\{0.5\}$ & $\{0.6,0.7\}$ & $\{0.3\}$ & $\{0.3\}$ \\
\hline
\end{tabular}

\subsection{Operations on Hesitant Fuzzy Soft Sets}

Definition 17. The complement of a hesitant fuzzy soft set $(\widetilde{F}, A)$ is denoted by $(\widetilde{F}, A)^{c}$ and is defined by

$$
(\widetilde{F}, A)^{c}=\left(\widetilde{F}^{c}, A\right),
$$

where $\widetilde{F}^{c}: A \rightarrow \widetilde{H}(U)$ is a mapping given by $\widetilde{F}^{c}(e)=(\widetilde{F}(e))^{c}$ for all $e \in A$.

Clearly, $\left(\widetilde{F}^{c}\right)^{c}$ is the same as $\widetilde{F}$ and $\left((\widetilde{F}, A)^{c}\right)^{c}=(\widetilde{F}, A)$.

It is worth noting that in the above definition of complement, the parameter set of the complement $(\widetilde{F}, A)^{c}$ is still the original parameter set $A$, instead of $\neg A$.

Example 18. Reconsider Example 11; the $(\widetilde{F}, A)^{c}$ can be calculated as follows:

$$
\begin{aligned}
\widetilde{F}^{c}\left(e_{1}\right)= & \left\{\frac{h_{1}}{\{0.7,0.8\}}, \frac{h_{2}}{\{0.4,0.5\}}, \frac{h_{3}}{\{0.7\}}, \frac{h_{4}}{\{0.5,0.7\}},\right. \\
& \left.\frac{h_{5}}{\{0.5,0.6\}}, \frac{h_{6}}{\{0.3,0.4\}}\right\}, \\
\widetilde{F}^{c}\left(e_{2}\right)= & \left\{\frac{h_{1}}{\{0.3,0.4,0.6\}}, \frac{h_{2}}{\{0.2,0.3,0.5\}}, \frac{h_{3}}{\{0.2,0.4\}},\right. \\
\widetilde{F}^{c}\left(e_{3}\right)= & \left\{\frac{h_{4}}{\{0.1,0.3\}}, \frac{h_{1}}{\{0.6,0.8\}}, \frac{h_{5}}{\{0.3,0.4\}}, \frac{h_{3}}{\{0.1,0.2\}}, \frac{h_{6}}{\{0.5,0.7\}},\right. \\
& \left.\frac{h_{5}}{\{0.4,0.6\}}, \frac{h_{6}}{\{0.3\}}\right\}, \\
\widetilde{F}^{c}\left(e_{4}\right)= & \left\{\frac{h_{1}}{\{0.4,0.5,0.7\}}, \frac{h_{2}}{\{0.8\}}, \frac{h_{3}}{\{0.5\}}, \frac{h_{4}}{\{0.3,0.4\}},\right. \\
& \left.\frac{h_{5}}{\{0.4,0.5\}}, \frac{h_{6}}{\{0.2\}}\right\}, \\
\widetilde{F}^{c}\left(e_{5}\right)= & \left\{\frac{h_{1}}{\{0.4\}}, \frac{h_{5}}{\{0.5,0.5\}}, \frac{h_{2}}{\{0.5,0.7\}}\right\} \cdot, \frac{h_{3}}{\{0.3,0.5\}}, \frac{h_{4}}{\{0.6,0.8\}},
\end{aligned},
$$

Definition 19. The AND operation on two hesitant fuzzy soft sets $(\widetilde{F}, A)$ and $(\widetilde{G}, B)$ which is denoted by $(\widetilde{F}, A) \wedge(\widetilde{G}, B)$ is defined by $(\widetilde{F}, A) \wedge(\widetilde{G}, B)=(\widetilde{J}, A \times B)$, where $\widetilde{J}(\alpha, \beta)=\widetilde{F}(\alpha) \cap$ $\widetilde{G}(\beta)$, for all $(\alpha, \beta) \in A \times B$.

Definition 20. The OR operation on the two hesitant fuzzy soft sets $(\widetilde{F}, A)$ and $(\widetilde{G}, B)$ which is denoted by $(\widetilde{F}, A) \vee(\widetilde{G}, B)$ is defined by $(\widetilde{F}, A) \vee(\widetilde{G}, B)=(\widetilde{O}, A \times B)$, where $\widetilde{O}(\alpha, \beta)=$ $\widetilde{F}(\alpha) \cup \widetilde{G}(\beta)$, for all $(\alpha, \beta) \in A \times B$.

Example 21. The results of "AND" and "OR" operations on the hesitant fuzzy soft sets $(\widetilde{F}, A)$ and $(\widetilde{G}, B)$ in Example 13 are shown in Tables 4 and 5 , respectively.

Theorem 22 (De Morgan's laws of hesitant fuzzy soft sets). Let $(\widetilde{F}, A)$ and $(\widetilde{G}, B)$ be two hesitant fuzzy soft sets over $U$; we have

$$
\begin{aligned}
& \text { (1) }((\widetilde{F}, A) \wedge(\widetilde{G}, B))^{c}=(\widetilde{F}, A)^{c} \vee(\widetilde{G}, B)^{c}, \\
& \text { (2) }((\widetilde{F}, A) \vee(\widetilde{G}, B))^{c}=(\widetilde{F}, A)^{c} \wedge(\widetilde{G}, B)^{c} .
\end{aligned}
$$

Proof. (1) Suppose that $(\widetilde{F}, A) \wedge(\widetilde{G}, B)=(\widetilde{J}, A \times B)$. Therefore $((\widetilde{F}, A) \wedge(\widetilde{G}, B))^{c}=(\widetilde{J}, A \times B)^{c}=\left(\widetilde{J}^{c}, A \times B\right)$. Similarly, $(\widetilde{F}, A)^{c} \vee(\widetilde{G}, B)^{c}=\left(\widetilde{F}^{c}, A\right) \vee\left(\widetilde{G}^{c}, B\right)=(\widetilde{O}, A \times B)$. Now take $(\alpha, \beta) \in A \times B$; therefore,

$$
\begin{aligned}
\widetilde{J}^{c}(\alpha, \beta) & (\widetilde{J}(\alpha, \beta))^{c}=(F(\alpha) \cap G(\beta))^{c} \\
= & \left\{\left\langle u, h_{\widetilde{A}}(u) \cap h_{\widetilde{B}}(u)\right\rangle \mid u \in U\right\}^{c} \\
= & \left\{\left\langle u,\left\{h \in\left(h_{\widetilde{A}} \cup h_{\widetilde{B}}\right) \mid h \leq \min \left(h_{\widetilde{A}}^{+}, h_{\widetilde{B}}^{+}\right)\right\}\right\rangle \mid u \in U\right\}^{c} \\
= & \left\{\left\langleu,\left\{1-h \mid h \in\left(h_{\widetilde{A}} \cup h_{\widetilde{B}}\right)\right.\right.\right. \\
& \left.\left.\left.\wedge h \leq \min \left(h_{\widetilde{A}}^{+}, h_{\widetilde{B}}^{+}\right)\right\}\right\rangle \mid u \in U\right\} \\
= & \left\{\left\langleu,\left\{1-h \mid(1-h) \in\left(1-h_{\widetilde{A}}\right) \cup\left(1-h_{\widetilde{B}}\right)\right.\right.\right. \\
& \wedge(1-h) \geq \max \left(\left(1-h_{\widetilde{A}}\right)^{-},\right. \\
= & \left.\left.\left.\left.\quad\left(1-h_{\widetilde{B}}\right)^{-}\right)\right\}\right\rangle \mid u \in U\right\} \\
& \left.\left.\left.\quad \geq \max \left(\left(1-h_{\widetilde{A}}\right)^{-},\left(1-h_{\widetilde{B}}\right)^{-}\right)\right\}\right\rangle \mid u \in U\right\},
\end{aligned}
$$


TABLE 5: The result of “OR" operation on $(\widetilde{F}, A)$ and $(\widetilde{G}, B)$.

\begin{tabular}{|c|c|c|c|c|c|c|}
\hline$U$ & $e_{1}, e_{1}$ & $e_{1}, e_{2}$ & $e_{1}, e_{3}$ & $e_{2}, e_{1}$ & $e_{2}, e_{2}$ & $e_{2}, e_{3}$ \\
\hline$h_{1}$ & $\{0.3\}$ & $\{0.4,0.5,0.6,0.7\}$ & $\{0.2,0.3,0.4\}$ & $\{0.4,0.6,0.7\}$ & $\{0.4,0.5,0.6,0.7\}$ & $\{0.4,0.6,0.7\}$ \\
\hline$h_{2}$ & $\{0.6,0.8\}$ & $\{0.7,0.8\}$ & $\{0.6,0.7\}$ & $\{0.6,0.7,0.8\}$ & $\{0.7,0.8\}$ & $\{0.6,0.7,0.8\}$ \\
\hline$h_{3}$ & $\{0.4,0.5\}$ & $\{0.9\}$ & $\{0.8,0.9\}$ & $\{0.6,0.8\}$ & $\{0.9\}$ & $\{0.8,0.9\}$ \\
\hline$h_{4}$ & $\{0.3,0.4,0.5\}$ & $\{0.8,0.9\}$ & $\{0.3,0.5\}$ & $\{0.7,0.9\}$ & $\{0.8,0.9\}$ & $\{0.7,0.9\}$ \\
\hline$h_{5}$ & $\{0.5\}$ & $\{0.5\}$ & $\{0.4,0.5,0.6\}$ & $\{0.5\}$ & $\{0.5\}$ & $\{0.4,0.5,0.6\}$ \\
\hline$h_{6}$ & $\{0.7\}$ & $\{0.6,0.7\}$ & $\{0.7\}$ & $\{0.7\}$ & $\{0.5\}$ & $\{0.7\}$ \\
\hline
\end{tabular}

$$
\begin{aligned}
\widetilde{O} & (\alpha, \beta) \\
& =F^{c}(\alpha) \cup G^{c}(\beta) \\
& =\left\{\left\langle u, h_{\widetilde{A}}(u)\right\rangle \mid u \in U\right\}^{c} \cup\left\{\left\langle u, h_{\widetilde{B}}(u)\right\rangle \mid u \in U\right\}^{c} \\
& =\left\{\left\langle u, 1-h_{\widetilde{A}}(u)\right\rangle \mid u \in U\right\} \cup\left\{\left\langle u, 1-h_{\widetilde{B}}(u)\right\rangle \mid u \in U\right\} \\
& =\left\{\left\langle u,\left(1-h_{\widetilde{A}}\right) \cup\left(1-h_{\widetilde{B}}\right)\right\rangle \mid u \in U\right\} \\
& =\left\{\left\langleu,\left\{(1-h) \in\left(1-h_{\widetilde{A}}\right) \cup\left(1-h_{\widetilde{B}}\right) \mid(1-h)\right.\right.\right. \\
& \left.\left.\left.\quad \geq \max \left(\left(1-h_{\widetilde{A}}\right)^{-},\left(1-h_{\widetilde{B}}\right)^{-}\right)\right\}\right\rangle \mid u \in U\right\} .
\end{aligned}
$$

Hence, $\widetilde{J}^{c}(\alpha, \beta)=\widetilde{O}(\alpha, \beta)$ is proved.

(2) Similar to the above process, it is easy to prove that $((\widetilde{F}, A) \vee(\widetilde{G}, B))^{c}=(\widetilde{F}, A)^{c} \wedge(\widetilde{G}, B)^{c}$.

Theorem 23. Let $(\widetilde{F}, A),(\widetilde{G}, B)$, and $(\widetilde{J}, C)$ be three hesitant fuzzy soft sets over $U$. Then the associative law of hesitant fuzzy soft sets holds as follows:

$$
\begin{aligned}
& (\widetilde{F}, A) \wedge((\widetilde{G}, B) \wedge(\widetilde{J}, C))=((\widetilde{F}, A) \wedge(\widetilde{G}, B)) \wedge(\widetilde{J}, C) \\
& (\widetilde{F}, A) \vee((\widetilde{G}, B) \vee(\widetilde{J}, C))=((\widetilde{F}, A) \vee(\widetilde{G}, B)) \vee(\widetilde{J}, C) .
\end{aligned}
$$

Proof. For all $\alpha \in A, \beta \in B$, and $\delta \in C$, because $\widetilde{F}(\alpha) \cap$ $(\widetilde{G}(\beta) \cap \widetilde{J}(\delta))=(\widetilde{F}(\alpha) \cap \widetilde{G}(\beta)) \cap \widetilde{J}(\delta)$, we can conclude that $(\widetilde{F}, A) \wedge((\widetilde{G}, B) \wedge(\widetilde{J}, C))=((\widetilde{F}, A) \wedge(\widetilde{G}, B)) \wedge(\widetilde{J}, C)$ holds.

Similarly, we can also conclude that $(\widetilde{F}, A) \vee((\widetilde{G}, B) \vee$ $(\widetilde{J}, C))=((\widetilde{F}, A) \vee(\widetilde{G}, B)) \vee(\widetilde{J}, C)$.

Remark 24. The distribution law of hesitant fuzzy soft sets does not hold. That is,

$$
\begin{aligned}
& (\widetilde{F}, A) \wedge((\widetilde{G}, B) \vee(\widetilde{J}, C)) \\
& \quad \neq((\widetilde{F}, A) \wedge(\widetilde{J}, C)) \vee((\widetilde{G}, B) \wedge(\widetilde{J}, C)), \\
& (\widetilde{F}, A) \vee((\widetilde{G}, B) \wedge(\widetilde{J}, C)) \\
& \quad \neq((\widetilde{F}, A) \vee(\widetilde{J}, C)) \wedge((\widetilde{G}, B) \vee(\widetilde{J}, C)) .
\end{aligned}
$$

For all $\alpha \in A, \beta \in B$, and $\delta \in C$, because $\widetilde{F}(\alpha) \cap(\widetilde{G}(\beta) \cup$ $\widetilde{J}(\delta)) \neq(\widetilde{F}(\alpha) \cap \widetilde{G}(\beta)) \cup(\widetilde{F}(\alpha) \cap \widetilde{J}(\delta))$ for the hesitant fuzzy sets, we can conclude that $(\widetilde{F}, A) \wedge((\widetilde{G}, B) \vee(\widetilde{J}, C)) \neq((\widetilde{F}, A)$
TABLE 6: The Union of hesitant fuzzy soft sets $(\widetilde{F}, A)$ and $(\widetilde{G}, B)$.

\begin{tabular}{cccc}
\hline$U$ & $e_{1}$ & $e_{2}$ & $e_{3}$ \\
\hline$h_{1}$ & $\{0.3\}$ & $\{0.4,0.5,0.6,0.7\}$ & $\{0.2,0.4\}$ \\
$h_{2}$ & $\{0.6,0.8\}$ & $\{0.7,0.8\}$ & $\{0.6,0.7\}$ \\
$h_{3}$ & $\{0.4,0.5\}$ & $\{0.9\}$ & $\{0.8,0.9\}$ \\
$h_{4}$ & $\{0.3,0.4,0.5\}$ & $\{0.8,0.9\}$ & $\{0.3,0.5\}$ \\
$h_{5}$ & $\{0.5\}$ & $\{0.5\}$ & $\{0.4,0.6\}$ \\
$h_{6}$ & $\{0.7\}$ & $\{0.5\}$ & $\{0.7\}$ \\
\hline
\end{tabular}

$\wedge(\widetilde{J}, C)) \vee((\widetilde{G}, B) \wedge(\widetilde{J}, C))$. Similarly, we can also conclude that $(\widetilde{F}, A) \vee((\widetilde{G}, B) \wedge(\widetilde{J}, C)) \neq((\widetilde{F}, A) \vee(\widetilde{J}, C)) \wedge((\widetilde{G}, B) \vee(\widetilde{J}, C))$

Definition 25. Union of two hesitant fuzzy soft sets $(\widetilde{F}, A)$ and $(\widetilde{G}, B)$ over $U$ is the hesitant fuzzy soft set $(\widetilde{J}, C)$, where $C=$ $A \cup B$, and for all $e \in C$,

$$
\begin{aligned}
\widetilde{J}(e) & =\widetilde{F}(e), \quad \text { if } e \in A-B, \\
& =\widetilde{G}(e), \quad \text { if } e \in B-A, \\
& =\widetilde{F}(e) \cup \widetilde{G}(e), \quad \text { if } e \in A \cap B .
\end{aligned}
$$

We write $(\widetilde{F}, A) \widetilde{U}(\widetilde{G}, B)=(\widetilde{J}, C)$.

Example 26. Reconsider Example 13, the Union of hesitant fuzzy soft sets $(\widetilde{F}, A)$ and $(\widetilde{G}, B)$ is shown in Table 6 .

Definition 27. Intersection of two hesitant fuzzy soft sets $(\widetilde{F}, A)$ and $(\widetilde{G}, B)$ with $A \cap B \neq \phi$ over $U$ is the hesitant fuzzy soft set $(\widetilde{J}, C)$, where $C=A \cap B$, and for all $e \in C, \widetilde{J}(e)=$ $\widetilde{F}(e) \cap \widetilde{G}(e)$.

$$
\text { We write }(\widetilde{F}, A) \widetilde{\cap}(\widetilde{G}, B)=(\widetilde{J}, C) \text {. }
$$

Example 28. Reconsider Example 13, the Intersection of hesitant fuzzy soft sets $(\widetilde{F}, A)$ and $(\widetilde{G}, B)$ is shown in Table 7 .

Theorem 29. Let $(\widetilde{F}, A)$ and $(\widetilde{G}, B)$ be two hesitant fuzzy soft sets over $U$. Then,
(1) $(\widetilde{F}, A) \widetilde{\cup}(\widetilde{F}, A)=(\widetilde{F}, A)$,
(2) $(\widetilde{F}, A) \widetilde{\cap}(\widetilde{F}, A)=(\widetilde{F}, A)$,
(3) $(\widetilde{F}, A) \cup \widetilde{\Phi}_{A}=(\widetilde{F}, A)$,
(4) $(\widetilde{F}, A) \cap \widetilde{\Phi}_{A}=\widetilde{\Phi}_{A}$,
(5) $(\widetilde{F}, A) \cup \widetilde{U}_{A}=\widetilde{U}_{A}$, 
TABLE 7: The Intersection of hesitant fuzzy soft sets $(\widetilde{F}, A)$ and $(\widetilde{G}, B)$.

\begin{tabular}{lcc}
\hline$U$ & $e_{1}$ & $e_{2}$ \\
\hline$h_{1}$ & $\{0.2,0.3\}$ & $\{0.4,0.5,0.6,0.7\}$ \\
$h_{2}$ & $\{0.5,0.7,0.8\}$ \\
$h_{3}$ & $\{0.5,0.6\}$ & $\{0.6,0.8\}$ \\
$h_{4}$ & $\{0.3\}$ & $\{0.7,0.8,0.9\}$ \\
$h_{5}$ & $\{0.3,0.5\}$ & $\{0.3,0.4,0.5\}$ \\
$h_{6}$ & $\{0.3,0.4,0.5\}$ & $\{0.3\}$ \\
\hline
\end{tabular}

(6) $(\widetilde{F}, A) \cap \widetilde{U}_{A}=(\widetilde{F}, A)$,

(7) $(\widetilde{F}, A) \widetilde{\cup}(\widetilde{G}, B)=(\widetilde{G}, B) \widetilde{\cup}(\widetilde{F}, A)$,

(8) $(\widetilde{F}, A) \widetilde{\cap}(\widetilde{G}, B)=(\widetilde{G}, B) \widetilde{\cap}(\widetilde{F}, A)$.

Theorem 30. Let $(\widetilde{F}, A)$ and $(\widetilde{G}, B)$ be two hesitant fuzzy soft sets over $U$. Then,

(1) $((\widetilde{F}, A) \widetilde{U}(\widetilde{G}, B))^{c} \widetilde{C}(\widetilde{F}, A)^{c} \widetilde{U}(\widetilde{G}, B)^{c}$,

(2) $(\widetilde{F}, A)^{c} \widetilde{\cap}(\widetilde{G}, B)^{c} \widetilde{c}((\widetilde{F}, A) \widetilde{\cap}(\widetilde{G}, B))^{c}$.

Proof. (1) Suppose that $(\widetilde{F}, A) \widetilde{U}(\widetilde{G}, B)=(\widetilde{J}, C)$, where $C=$ $A \cup B$, and for all $e \in C$,

$$
\begin{aligned}
\widetilde{J}(e) & =\widetilde{F}(e), \quad \text { if } e \in A-B, \\
& =\widetilde{G}(e), \quad \text { if } e \in B-A, \\
& =\widetilde{F}(e) \cup \widetilde{G}(e), \quad \text { if } e \in A \cap B .
\end{aligned}
$$

Thus $((\widetilde{F}, A) \cup(\widetilde{G}, B))^{c}=(\widetilde{J}, C)^{c}=\left(\widetilde{J}^{c}, C\right)$, and

$$
\begin{aligned}
\widetilde{J}^{c}(e) & =\widetilde{F}^{c}(e), \quad \text { if } e \in A-B, \\
& =\widetilde{G}^{c}(e), \quad \text { if } e \in B-A, \\
& =\widetilde{F}^{c}(e) \cap \widetilde{G}^{c}(e), \quad \text { if } e \in A \cap B .
\end{aligned}
$$

Similarly, suppose that $(\widetilde{F}, A)^{c} \widetilde{\cup}(\widetilde{G}, B)^{c}=\left(\widetilde{F}^{c}, A\right) \widetilde{\cup}\left(\widetilde{G}^{c}, B\right)=$ $(\widetilde{O}, C)$, where $C=A \cup B$, and for all $e \in C$,

$$
\begin{aligned}
\widetilde{O}(e) & =\widetilde{F}^{c}(e), \quad \text { if } e \in A-B, \\
& =\widetilde{G}^{c}(e), \quad \text { if } e \in B-A, \\
& =\widetilde{F}^{c}(e) \cup \widetilde{G}^{c}(e), \quad \text { if } e \in A \cap B .
\end{aligned}
$$

Obviously, for all $e \in C, \widetilde{J}^{c}(e) \widetilde{\subseteq} \widetilde{O}(e)$. Part (1) of Theorem 30 is proved.

(2) Similar to the above process, it is easy to prove that $(\widetilde{F}, A)^{c} \widetilde{\cap}(\widetilde{G}, B)^{c} \widetilde{C}((\widetilde{F}, A) \widetilde{\cap}(\widetilde{G}, B))^{c}$.

Theorem 31. Let $(\widetilde{F}, A)$ and $(\widetilde{G}, B)$ be two hesitant fuzzy soft sets over $U$. Then,

(1) $(\widetilde{F}, A)^{c} \widetilde{\cap}(\widetilde{G}, B)^{c} \widetilde{\subset}((\widetilde{F}, A) \widetilde{U}(\widetilde{G}, B))^{c}$,

(2) $((\widetilde{F}, A) \widetilde{\cap}(\widetilde{G}, B))^{c} \widetilde{C}(\widetilde{F}, A)^{c} \widetilde{\cup}(\widetilde{G}, B)^{c}$.
Proof. (1) Suppose that $(\widetilde{F}, A) \widetilde{\cup}(\widetilde{G}, B)=(\widetilde{J}, C)$, where $C=$ $A \cup B$, and for all $e \in C$,

$$
\begin{aligned}
\widetilde{J}(e) & =\widetilde{F}(e), \quad \text { if } e \in A-B, \\
& =\widetilde{G}(e), \quad \text { if } e \in B-A, \\
& =\widetilde{F}(e) \cup \widetilde{G}(e), \quad \text { if } e \in A \cap B .
\end{aligned}
$$

Then $((\widetilde{F}, A) \cup(\widetilde{G}, B))^{c}=(\widetilde{J}, C)^{c}=\left(\widetilde{J}^{c}, C\right)$, and

$$
\begin{aligned}
\widetilde{J}^{c}(e) & =\widetilde{F}^{c}(e), \quad \text { if } e \in A-B, \\
& =\widetilde{G}^{c}(e), \quad \text { if } e \in B-A, \\
& =\widetilde{F}^{c}(e) \cap \widetilde{G}^{c}(e), \quad \text { if } e \in A \cap B .
\end{aligned}
$$

Similarly, suppose $(\widetilde{F}, A)^{c} \widetilde{\cap}(\widetilde{G}, B)^{c}=\left(\widetilde{F}^{c}, A\right) \widetilde{\cap}\left(\widetilde{G}^{c}, B\right)=(\widetilde{O}$, $J)$, where $J=A \cap B$. Then, for all $e \in J, \widetilde{O}(e)=F^{c}(e) \cap G^{c}(e)$.

Hence, it is obvious that $J \subseteq C$ and, for all $e \in J, \widetilde{O}(e)=$ $\widetilde{J}^{c}(e)$; thus, $(\widetilde{F}, A)^{c} \widetilde{\cap}(\widetilde{G}, B)^{c} \widetilde{C}((\widetilde{F}, A) \widetilde{\cup}(\widetilde{G}, B))^{c}$ is proved.

(2) Similar to the above process, it is easy to prove that $((\widetilde{F}, A) \widetilde{\cap}(\widetilde{G}, B))^{c} \widetilde{c}(\widetilde{F}, A)^{c} \widetilde{U}(\widetilde{G}, B)^{c}$.

Theorem 32. Let $(\widetilde{F}, A)$ and $(\widetilde{G}, A)$ be two hesitant fuzzy soft sets over $U$; then,
(1) $((\widetilde{F}, A) \widetilde{U}(\widetilde{G}, A))^{c}=(\widetilde{F}, A)^{c} \widetilde{\cap}(\widetilde{G}, A)^{c}$,
(2) $((\widetilde{F}, A) \widetilde{\cap}(\widetilde{G}, A))^{c}=(\widetilde{F}, A)^{c} \widetilde{U}(\widetilde{G}, A)^{c}$.

Proof. (1) Suppose that $(\widetilde{F}, A) \widetilde{U}(\widetilde{G}, A)=(\widetilde{J}, A)$, and for all $e \in A$,

$$
\widetilde{J}(e)=\widetilde{F}(e) \cup \widetilde{G}(e) .
$$

Then $((\widetilde{F}, A) \cup(\widetilde{G}, A))^{c}=(\widetilde{J}, A)^{c}=\left(\widetilde{J}^{c}, A\right)$, and for all $e \in A$,

$$
\widetilde{J}^{c}(e)=\widetilde{F}^{c}(e) \cap \widetilde{G}^{c}(e) .
$$

Similarly, suppose that $(\widetilde{F}, A)^{c} \widetilde{\cap}(\widetilde{G}, A)^{c}=\left(\widetilde{F}^{c}, A\right) \widetilde{\cap}\left(\widetilde{G}^{c}, A\right)=$ $(\widetilde{O}, A)$, and for all $e \in A$,

$$
\widetilde{O}(e)=\widetilde{F}^{c}(e) \cap \widetilde{G}^{c}(e) .
$$

Hence, $\widetilde{O}(e)=\widetilde{J}^{c}(e)$; the $((\widetilde{F}, A) \widetilde{U}(\widetilde{G}, A))^{c}=(\widetilde{F}, A)^{c} \widetilde{\cap}(\widetilde{G}, A)^{c}$ is proved.

(2) Similar to the above process, it is easy to prove that $((\widetilde{F}, A) \widetilde{\cap}(\widetilde{G}, A))^{c}=(\widetilde{F}, A)^{c} \widetilde{\cup}(\widetilde{G}, A)^{c}$.

\section{Application of Hesitant Fuzzy Soft Sets}

Roy and Maji [14] presented an algorithm to solve the decision making problems according to a comparison table from the fuzzy soft set. Later, Kong et al. [15] pointed out that the algorithm in Roy and Maji [14] was incorrect by giving a counterexample. Kong et al. [15] also presented a modified algorithm which was based on the comparison of choice values of different objects [13]. However, Feng et al. [16] further explored the fuzzy soft set based decision making problems 
TABLE 8: The tabular representation of the hesitant fuzzy soft set $(\widetilde{F}, A)$ in Example 35.

\begin{tabular}{|c|c|c|c|c|c|}
\hline$U$ & $e_{1}$ & $e_{2}$ & $e_{3}$ & $e_{4}$ & $e_{5}$ \\
\hline$x_{1}$ & $\{0.5,0.6\}$ & $\{0.3,0.5\}$ & $\{0.3\}$ & $\{0.3,0.4\}$ & $\{0.6,0.9\}$ \\
\hline$x_{2}$ & $\{0.7\}$ & $\{0.4\}$ & $\{0.2,0.4,0.5\}$ & $\{0.4,0.5\}$ & $\{0.5,0.6\}$ \\
\hline$x_{3}$ & $\{0.4,0.6\}$ & $\{0.6,0.7\}$ & $\{0.5,0.6\}$ & $\{0.6\}$ & $\{0.2,0.3,0.5\}$ \\
\hline$x_{4}$ & $\{0.3,0.5\}$ & $\{0.4,0.6\}$ & $\{0.5\}$ & $\{0.6,0.7\}$ & $\{0.2,0.4,0.5\}$ \\
\hline$x_{5}$ & $\{0.5,0.6,0.8\}$ & $\{0.3\}$ & $\{0.3,0.4\}$ & $\{0.2,0.3,0.5\}$ & $\{0.7,0.8\}$ \\
\hline
\end{tabular}

more deeply and pointed out that the concept of choice values was not suitable to solve the decision making problems involving fuzzy soft sets, though it was an efficient method to solve the crisp soft set based decision making problems. They proposed a novel approach by using the level soft sets to solve the fuzzy soft set based decision making problems. Thus, some decision making problems that cannot be solved by the methods in Roy and Maji [14] and Kong et al. [15] can be successfully solved by this approach. In the following, we will apply this level soft sets method to hesitant fuzzy soft set based decision making problems.

Let $U=\left\{u_{1}, u_{2}, \ldots, u_{n}\right\}$ and $(\widetilde{F}, A)$ be a hesitant fuzzy soft set over $U$. For every $e \in A, \widetilde{F}(e)=\left\{u_{1} / h_{\widetilde{A}}\left(u_{1}\right)\right.$, $\left.u_{2} / h_{\widetilde{A}}\left(u_{2}\right), \ldots, u_{n} / h_{\widetilde{A}}\left(u_{n}\right)\right\}$, we can calculate the score of each hesitant fuzzy element by Definition 7 . Thus, we define an induced fuzzy set with respect to $e$ in $U$ as $\mu_{\widetilde{F}(e)}=\left\{u_{1} /\right.$ $\left.s\left(h_{\widetilde{A}}\left(u_{1}\right)\right), u_{2} / s\left(h_{\widetilde{A}}\left(u_{2}\right)\right), \ldots, u_{n} / s\left(h_{\widetilde{A}}\left(u_{n}\right)\right)\right\}$. By using this method, we can change a hesitant fuzzy set $\widetilde{F}(e)$ into an induced fuzzy set $\mu_{\widetilde{F}(e)}$. Furthermore, once the induced fuzzy soft set of a hesitant fuzzy soft set has been obtained, we can determine the optimal alternative according to Feng's [16] algorithm. The explicit algorithm of the decision making based on hesitant fuzzy soft set is given as follows.

\section{Algorithm 33. Consider the following.}

(1) Input the hesitant fuzzy soft set $(\widetilde{F}, A)$.

(2) Compute the induced fuzzy soft set $\Delta_{\widetilde{F}}=(\widetilde{\Gamma}, A)$.

(3) Input a threshold fuzzy set $\lambda: A \rightarrow[0,1]$ (or give a threshold value $t \in[0,1]$; or choose the midlevel decision rule; or choose the top-level decision rule) for decision making.

(4) Compute the level soft set $L\left(\Delta_{\widetilde{F}} ; \lambda\right)$ of $\Delta_{\widetilde{F}}$ with respect to the threshold fuzzy set $\lambda$ (or the $t$-level soft set $L\left(\Delta_{\widetilde{F}} ; t\right)$; or the mid-level soft set $L\left(\Delta_{\widetilde{F}} ;\right.$ mid $)$; or the top-level soft set $\left.L\left(\Delta_{\widetilde{F}} ; \max \right)\right)$.

(5) Present the level soft set $L\left(\Delta_{\widetilde{F}} ; \lambda\right)$ (or $L\left(\Delta_{\widetilde{F}} ; t\right)$; or $L\left(\Delta_{\widetilde{F}} ;\right.$ mid); or $\left.L\left(\Delta_{\widetilde{F}} ; \max \right)\right)$ in tabular form and compute the choice value $c_{i}$ of $u_{i}$, for all $i$.

(6) The optimal decision is to select $u_{j}=\operatorname{argmax}_{i} c_{i}$.

(7) If there are more than one $u_{j}$ s, then any one of $u_{j}$ may be chosen.

Remark 34. In order to get a unique optimal choice according to the above algorithm, the decision makers can go back to
TABLE 9: The tabular representation of the induced fuzzy soft set $\Delta_{\widetilde{F}}=(\widetilde{\Gamma}, A)$ in Example 35 .

\begin{tabular}{lccccc}
\hline$U$ & $e_{1}$ & $e_{2}$ & $e_{3}$ & $e_{4}$ & $e_{5}$ \\
\hline$x_{1}$ & 0.55 & 0.4 & 0.3 & 0.35 & 0.75 \\
$x_{2}$ & 0.7 & 0.4 & 0.37 & 0.45 & 0.55 \\
$x_{3}$ & 0.5 & 0.65 & 0.55 & 0.6 & 0.33 \\
$x_{4}$ & 0.4 & 0.5 & 0.5 & 0.65 & 0.37 \\
$x_{5}$ & 0.63 & 0.3 & 0.35 & 0.33 & 0.75 \\
\hline
\end{tabular}

the third step and change the threshold (or decision criteria) in case that there is more than one optimal choice that can be obtained in the last step. Moreover, the final optimal decision can be adjusted according to the decision makers' preferences.

We adopt the following example to illustrate the idea of algorithm given above.

Example 35. Consider a retailer planning to open a new store in the city. There are five sites, $x_{i}(i=1,2, \ldots, 5)$, to be selected. Five attributes are considered: market $\left(e_{1}\right)$, traffic $\left(e_{2}\right)$, rent price $\left(e_{3}\right)$, competition $\left(e_{4}\right)$, and brand improvement $\left(e_{5}\right)$. Suppose that the retailer evaluates the optional five sites under various attributes with hesitant fuzzy element; then, hesitant fuzzy soft set $(\widetilde{F}, A)$ can describe the characteristics of the sites under the hesitant fuzzy information, which is shown in Table 8 .

In order to select the optimal sites to open the store based on the above hesitant fuzzy soft set, according to Algorithm 33, we can calculate the score of each hesitant fuzzy element and obtain the induced fuzzy soft set $\Delta_{\widetilde{F}}=(\widetilde{\Gamma}, A)$, which is shown as in Table 9.

As an adjustable approach, one can use different rules (or the thresholds) to get different decision results, according to his or her preference. For example, we use the midlevel decision rule in our paper and the midlevel threshold fuzzy set of $\Delta_{\widetilde{F}}$ is as $\operatorname{mid}_{\Delta_{\tilde{F}}}=\left\{\left(e_{1}, 0.556\right),\left(e_{2}, 0.45\right)\right.$, $\left.\left(e_{3}, 0.414\right),\left(e_{4}, 0.476\right),\left(e_{5}, 0.55\right)\right\}$. Furthering, we can get the midlevel soft set $L\left(\Delta_{\widetilde{F}} ;\right.$ mid $)$ of $\Delta_{\widetilde{F}}$, whose tabular form is as Table 10 shows. The choice values of each site are also shown in the last column of Table 10.

According to Table 10, the maximum choice value is 3 and so the optimal decision is to select $x_{3}$. Therefore, the retailer should select site 3 as the best site to open a store. 
TABLE 10: The tabular representation of the midlevel soft set $L\left(\Delta_{\widetilde{F}} ;\right.$ mid $)$ with choice values in Example 35 .

\begin{tabular}{ccccccc}
\hline$U$ & $e_{1}$ & $e_{2}$ & $e_{3}$ & $e_{4}$ & $e_{5}$ & Choice value \\
\hline$x_{1}$ & 0 & 0 & 0 & 0 & 1 & 1 \\
$x_{2}$ & 1 & 0 & 0 & 0 & 0 & 1 \\
$x_{3}$ & 0 & 1 & 1 & 1 & 0 & 3 \\
$x_{4}$ & 0 & 1 & 0 & 1 & 0 & 2 \\
$x_{5}$ & 1 & 0 & 0 & 0 & 1 & 2 \\
\hline
\end{tabular}

\section{Conclusion}

In this paper we consider the notion of hesitant fuzzy soft sets which combine the hesitant fuzzy sets and soft sets. Then we define the complement, "AND", "OR", union and intersection operations on hesitant fuzzy soft sets. The basic properties such as De Morgan's laws and the relevant laws of hesitant fuzzy soft sets are proved. Finally, we apply it to a decision making problem with the help of level soft set.

Our research can be explored deeply in two directions in the future. Firstly, we can combine other membership functions and soft sets to make novel soft sets which have different forms; Secondly, we can not only explore the application of hesitant fuzzy soft set in decision making more deeply, but also apply the hesitant fuzzy soft set in many other areas such as forecasting and data analysis.

\section{Conflict of Interests}

The authors declare that there is no conflict of interests regarding the publication of this paper.

\section{Acknowledgments}

This research is supported by the Innovation Group Science Foundation Project of the National Natural Science Foundation of China (no. 71221006), the Major International Collaboration Project of the National Natural Science Foundation of China (no. 71210003), and the Humanities and Social Sciences Major Project of the Ministry of Education of China (no. 13JZD016).

\section{References}

[1] D. Molodtsov, "Soft set theory-first results," Computers \& Mathematics with Applications, vol. 37, no. 4-5, pp. 19-31, 1999.

[2] P. K. Maji, R. Biswas, and A. R. Roy, "Fuzzy soft sets," Journal of Fuzzy Mathematics, vol. 9, no. 3, pp. 589-602, 2001.

[3] P. K. Maji, R. Biswas, and A. R. Roy, "Intuitionistic fuzzy soft sets," Journal of Fuzzy Mathematics, vol. 9, no. 3, pp. 677-692, 2001.

[4] P. K. Maji, A. R. Roy, and R. Biswas, "On intuitionistic fuzzy soft sets," Journal of Fuzzy Mathematics, vol. 12, no. 3, pp. 669-683, 2004.

[5] P. K. Maji, "More on intuitionistic fuzzy soft sets," in Proceedings of the 12th International Conference on Rough Sets, Fuzzy Sets, Data Mining and Granular Computing (RSFDGrC '09), H. Sakai, M. K. Chakraborty, A. E. Hassanien, D. Slezak, and W. Zhu,
Eds., vol. 5908 of Lecture Notes in Computer Science, pp. 231240, 2009.

[6] K. T. Atanassov, "Intuitionistic fuzzy sets," Fuzzy Sets and Systems, vol. 20, no. 1, pp. 87-96, 1986.

[7] K. T. Atanassov, Intuitionistic Fuzzy Sets, Physica-Verlag, New York, NY, USA, 1999.

[8] G. Deschrijver and E. E. Kerre, "On the relationship between some extensions of fuzzy set theory," Fuzzy Sets and Systems, vol. 133, no. 2, pp. 227-235, 2003.

[9] M. B. Gorzałczany, "A method of inference in approximate reasoning based on interval-valued fuzzy sets," Fuzzy Sets and Systems, vol. 21, no. 1, pp. 1-17, 1987.

[10] X. Yang, T. Y. Lin, J. Yang, Y. Li, and D. Yu, "Combination of interval-valued fuzzy set and soft set," Computers \& Mathematics with Applications, vol. 58, no. 3, pp. 521-527, 2009.

[11] Y. Jiang, Y. Tang, Q. Chen, H. Liu, and J. Tang, "Interval-valued intuitionistic fuzzy soft sets and their properties," Computers \& Mathematics with Applications, vol. 60, no. 3, pp. 906-918, 2010.

[12] Z. Xiao, S. Xia, K. Gong, and D. Li, "The trapezoidal fuzzy soft set and its application in MCDM," Applied Mathematical Modelling: Simulation and Computation for Engineering and Environmental Systems, vol. 36, no. 12, pp. 5844-5855, 2012.

[13] Y. Yang, X. Tan, and C. C. Meng, “The multi-fuzzy soft set and its application in decision making," Applied Mathematical Modelling: Simulation and Computation for Engineering and Environmental Systems, vol. 37, no. 7, pp. 4915-4923, 2013.

[14] A. R. Roy and P. K. Maji, "A fuzzy soft set theoretic approach to decision making problems," Journal of Computational and Applied Mathematics, vol. 203, no. 2, pp. 412-418, 2007.

[15] Z. Kong, L. Gao, and L. Wang, "Comment on "A fuzzy soft set theoretic approach to decision making problems"', Journal of Computational and Applied Mathematics, vol. 223, no. 2, pp. 540-542, 2009.

[16] F. Feng, Y. B. Jun, X. Liu, and L. Li, "An adjustable approach to fuzzy soft set based decision making," Journal of Computational and Applied Mathematics, vol. 234, no. 1, pp. 10-20, 2010.

[17] Y. Jiang, Y. Tang, and Q. Chen, "An adjustable approach to intuitionistic fuzzy soft sets based decision making," Applied Mathematical Modelling: Simulation and Computation for Engineering and Environmental Systems, vol. 35, no. 2, pp. 824-836, 2011.

[18] Y. B. Jun, “Soft BCK/BCI-algebras," Computers \& Mathematics with Applications, vol. 56, no. 5, pp. 1408-1413, 2008.

[19] Y. B. Jun and C. H. Park, "Applications of soft sets in ideal theory of BCK/BCI-algebras," Information Sciences, vol. 178, no. 11, pp. 2466-2475, 2008.

[20] Y. B. Jun, K. J. Lee, and C. H. Park, "Soft set theory applied to ideals in $d$-algebras," Computers \& Mathematics with Applications, vol. 57, no. 3, pp. 367-378, 2009.

[21] Y. B. Jun, K. J. Lee, and J. Zhan, "Soft p-ideals of soft BCIalgebras," Computers \& Mathematics with Applications, vol. 58, no. 10, pp. 2060-2068, 2009.

[22] F. Feng, X. Liu, V. L. Leoreanu-Fotea, and Y.B. Jun, "Soft sets and soft rough sets," Information Sciences: An International Journal, vol. 181, no. 6, pp. 1125-1137, 2011.

[23] F. Feng, C. Li, B. Davvaz, and M. I. Ali, "Soft sets combined with fuzzy sets and rough sets: a tentative approach," Soft Computing, vol. 14, no. 9, pp. 899-911, 2010.

[24] V. Torra and Y. Narukawa, "On hesitant fuzzy sets and decision," in Proceedings of the IEEE International Conference on Fuzzy Systems, pp. 1378-1382, Jeju-do, Republic of Korea, August 2009. 
[25] V. Torra, "Hesitant fuzzy sets," International Journal of Intelligent Systems, vol. 25, no. 6, pp. 529-539, 2010.

[26] Z. Zhang, "Hesitant fuzzy power aggregation operators and their application to multiple attribute group decision making," Information Sciences, vol. 234, pp. 150-181, 2013.

[27] M. Xia and Z. Xu, "Hesitant fuzzy information aggregation in decision making," International Journal of Approximate Reasoning, vol. 52, no. 3, pp. 395-407, 2011.

[28] Z. S. Xu and M. Xia, "Distance and similarity measures for hesitant fuzzy sets," Information Sciences, vol. 181, no. 11, pp. 2128-2138, 2011.

[29] H. Liu and G. Wang, "Multi-criteria decision-making methods based on intuitionistic fuzzy sets," European Journal of Operational Research, vol. 179, no. 1, pp. 220-233, 2007.

[30] J. M. Mendel, "Computing with words, when words can mean different things to different people," in Proceedings of the $3 \mathrm{rd}$ International ICSC Symposium on Fuzzy Logic and Applications, pp. 158-164, Rochester, NY, USA, 1999.

[31] J. M. Merigó and A. M. Gil-Lafuente, “The induced generalized OWA operator," Information Sciences, vol. 179, no. 6, pp. 729741, 2009. 


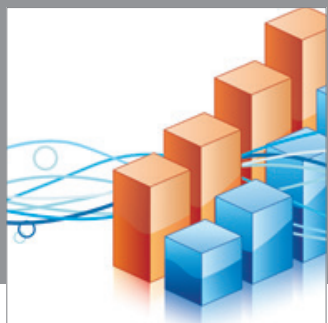

Advances in

Operations Research

mansans

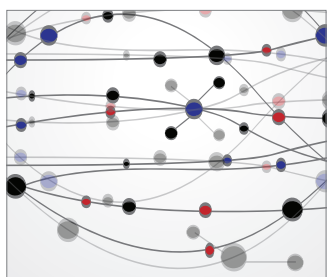

The Scientific World Journal
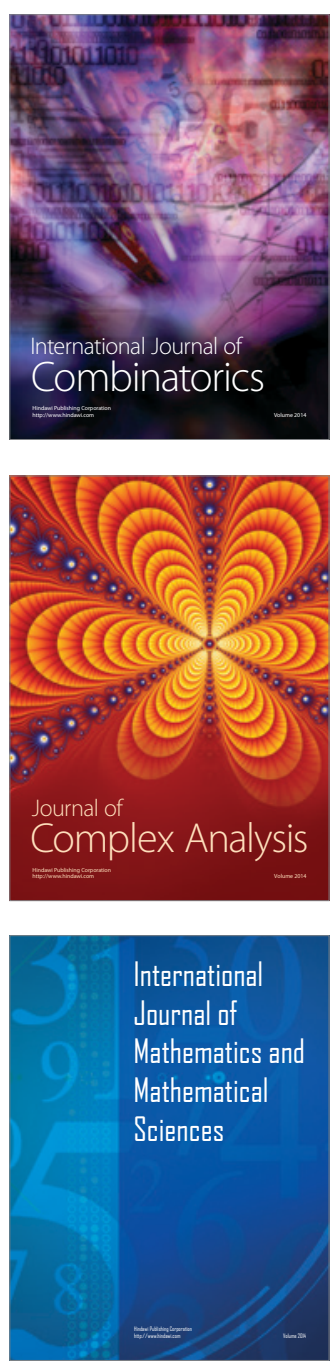
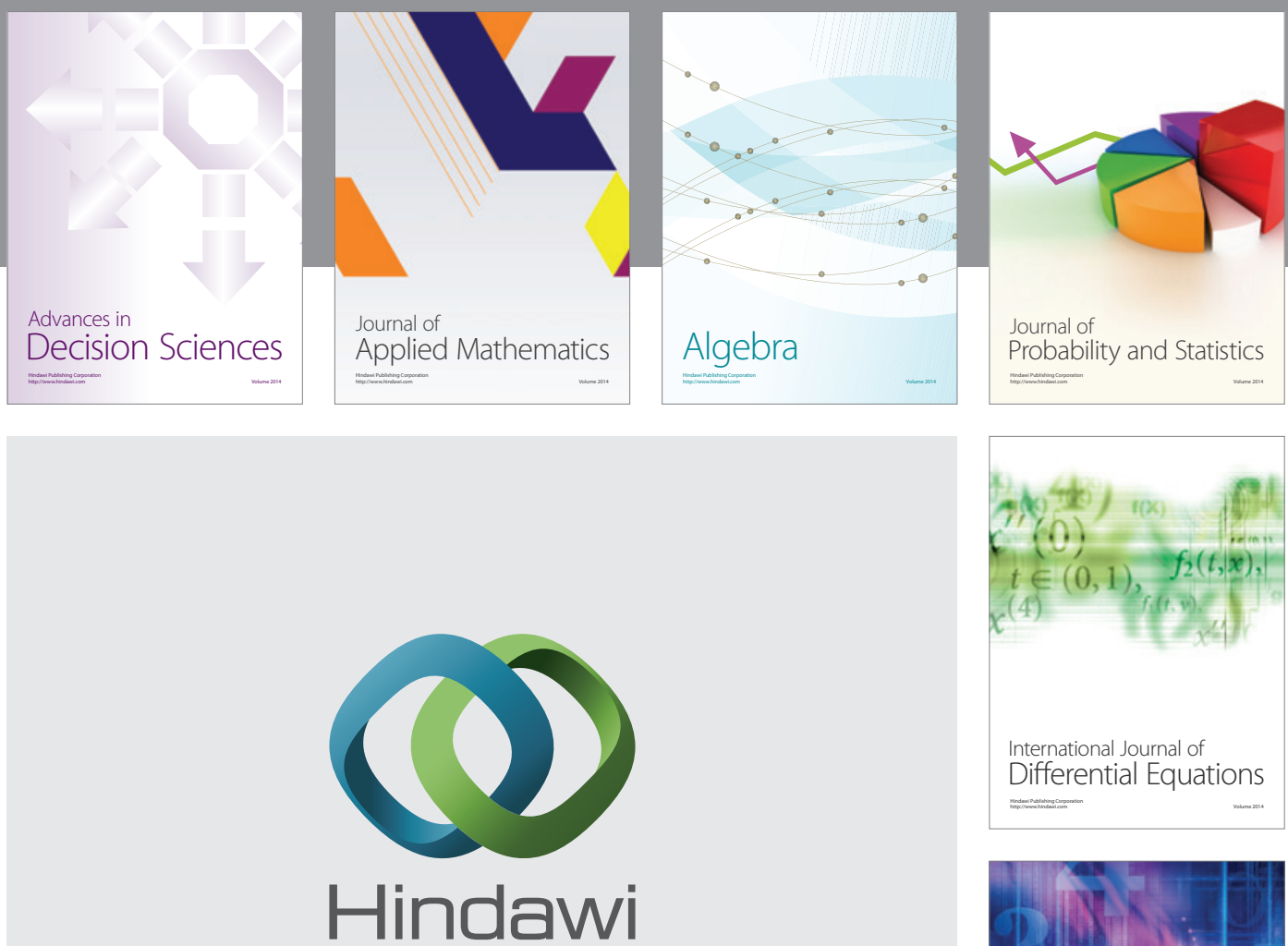

Submit your manuscripts at http://www.hindawi.com
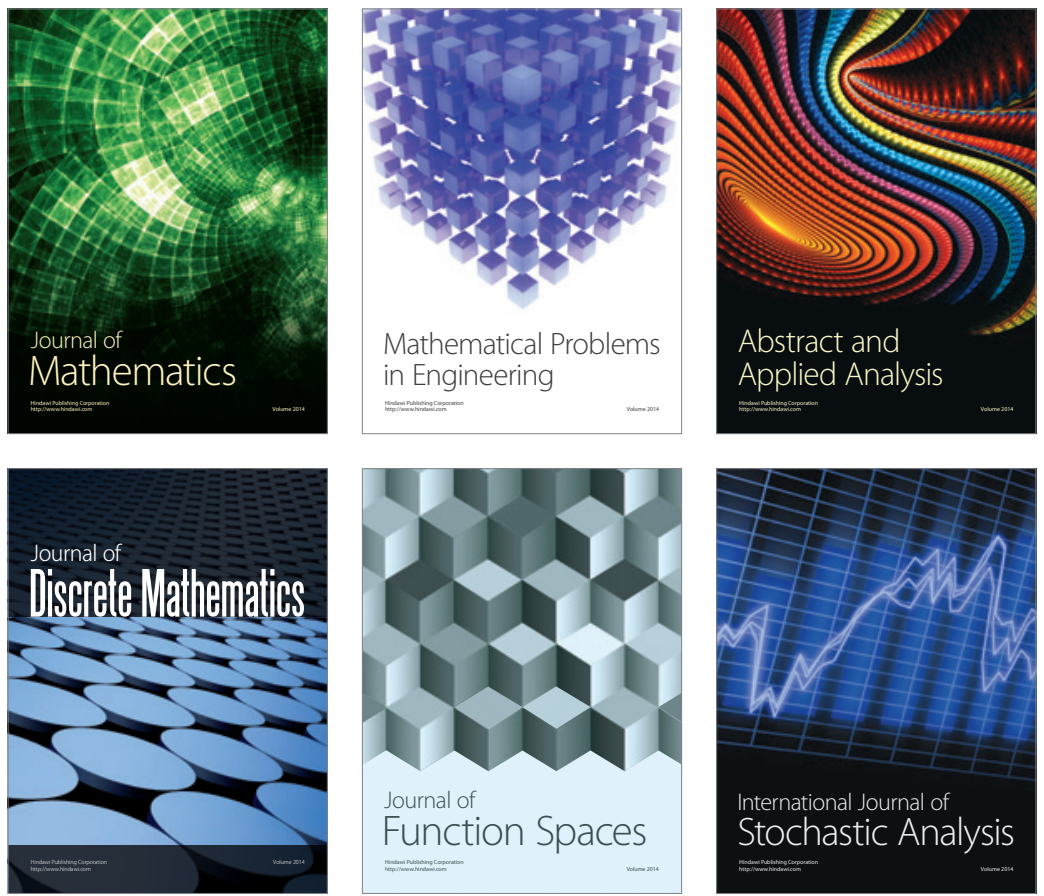

Journal of

Function Spaces

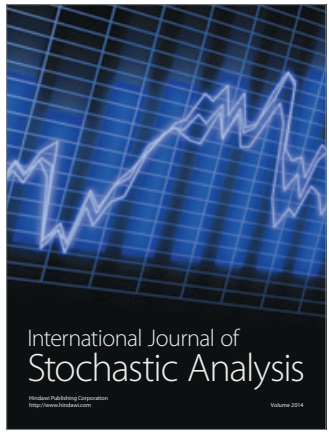

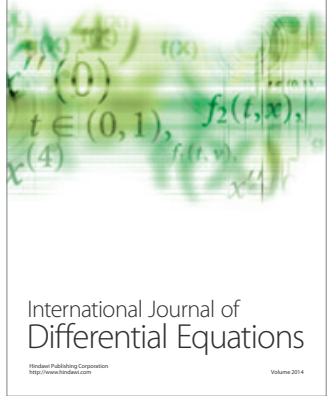
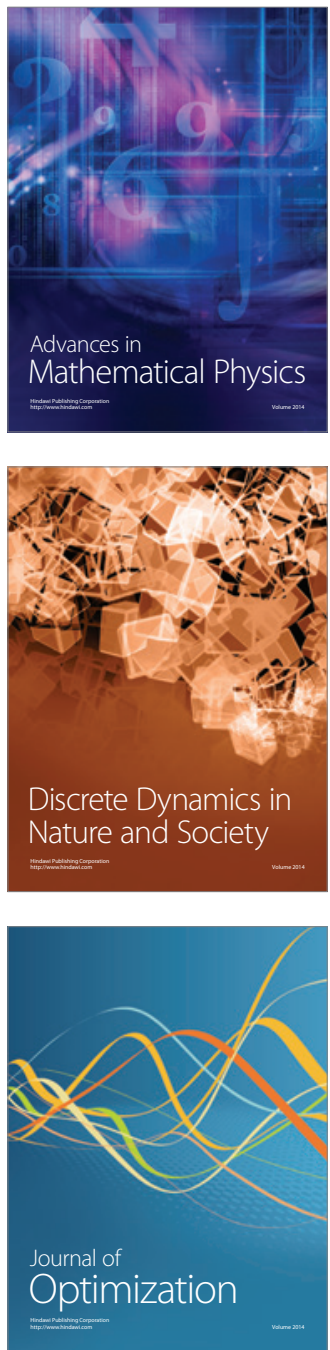